\title{
Surface photometry and structure of high redshift disk galaxies in the HDF-S NICMOS field
}

\author{
A. Tamm ${ }^{1,2}$ and P. Tenjes ${ }^{1,2}$ \\ ${ }^{1}$ Institute of Theoretical Physics, Tartu University, Tähe 4, Tartu, 51010, Estonia \\ 2 Tartu Observatory, Tõravere, Tartumaa, 61602 Estonia \\ e-mail: [atamm;ptenjes]@ut.ee
}

Received 18 August 2005 / Accepted 22 November 2005

\begin{abstract}
Aims. We report on a photometric study of a sample of 22 disk galaxies in the Hubble Deep Field South NICMOS parallel field. The redshift range of the galaxies is $z=0.5-2.6$.

Methods. We use deep NICMOS $J$ and $H$ band and STIS open mode images, taken as part of the HDF-S project, to construct rest-frame $B$-profiles and $(U-V)$ color profiles of the galaxies. Before fitting isophotes, images are deconvolved with PSF. Derived surface brightness profiles are approximated by Sérsic luminosity distribution.

Results. Significantly large population of disks cannot be represented by an exponential disk, but this can be well done by Sérsic law, if $n<1$. This might be the same phenomenon which has earlier been referred to as truncation of disks. Parameter $n$ does not vary significantly with redshift. Galactic sizes decrease with redshift as $r_{\mathrm{e}}(z) / r_{\mathrm{e}}(0)=1-0.26 z$. The rest frame $(U-V)$ color shows a clear decrease at $z \approx 2$, concordantly with the understanding of more intense star formation at earlier epochs. Color gradients $\Delta(U-V) / \Delta r$ are small and roughly constant at $z<2$. At $z>2$, dominantly positive gradients appear, possibly indicating centrally concentrated star-formation. On the basis of $(U-V)$ color and chemical evolution models, the disks observed at $z \sim 2.5$ have formed between $z=3.5-7$. Scale radii $r_{\mathrm{e}}$ of the galaxies correlate with the scale surface brightnesses $\mu_{\mathrm{e}}$ for the sample. None of the studied parameters shows clear dependence on absolute $B$ luminosity for the galaxies.
\end{abstract}

Key words. galaxies: photometry - galaxies: fundamental parameters - galaxies: high-redshift - galaxies: spiral - galaxies: structure

\section{Introduction}

Comparison of the structure of galactic disks at intermediate and high redshifts with the disks resulting from $\mathrm{N}$-body simulations allows us to test the corresponding algorithms and to specify physical processes involved in disk formation. General properties of both elliptical and disk galaxies are rather well reproduced by modeling (e.g. White \& Rees 1978; Navarro \& White 1994; Abadi et al. 2003; Bell et al. 2003; Governato et al. 2004; Robertson et al. 2004; Nagamine et al. 2005). The initial shortcomings have been corrected by now and it has become possible to model the formation of extended, rotationally supported disks. On the other hand, good models for calculating the formation and evolution of galaxies involve a great number of free parameters (see e.g. Cole et al. 2000).

To test and constrain the simulations carried out at increasing resolutions, detailed observational studies of individual high redshift galaxies are indispensable. In a number of papers, structural parameters of galaxies have been statistically studied at a wide range of redshifts. Luminosity function has been derived out to $z \sim 1.5-2$ by Chen et al. (2003) and Ilbert et al. (2005); to $z \sim 3.5$ by Poli et al. (2003) and Giallongo et al. (2005); to $z \sim 5$ by Gabasch et al. (2004). General morphologic studies and statistics out to redshift $z \approx 3$ and even further have been conducted by Bunker et al. (1999), van den Bergh et al. (2000), Corbin et al. (2000), Bouwens et al. (2004), Cassata et al. (2005) and many others.

Due to hierarchical clustering, galactic sizes should grow with time (e.g. Fall \& Efstathiou 1980). An analysis of galactic size distribution allows us to constrain certain parameters in galaxy evolution simulations (feedback etc., see Cole et al. 2000). The observations do not clearly confirm this prediction as yet. For example, galaxy size evolution with redshift has been studied by Ferguson et al. (2004) and Trujillo et al. (2004, 2005). They show that, at a given luminosity, decrease of the sizes of galaxies with redshift is clearly present. On the other hand, Ravindranath et al. (2004) and Cassata et al. (2005) do not detect significant evolution. Serious sources of controversies are selection effects and difficulties in conducting photometric analysis of galaxies at high redshifts, both caused 
Table 1. General information about the HDF-South NICMOS field observations.

\begin{tabular}{llllll}
\hline \hline Camera and filter & $\begin{array}{l}\text { Central } \\
\text { wavelength }(\mathrm{nm})\end{array}$ & $\begin{array}{l}\text { Bandwidth } \\
(F W H M, \mathrm{~nm})\end{array}$ & $\begin{array}{l}\text { Total exposure } \\
\text { time }(\mathrm{s})\end{array}$ & $\begin{array}{l}\text { PSF width } \\
\left(F W H M,{ }^{\prime \prime}\right)\end{array}$ & $\begin{array}{l}\text { Projected pixel } \\
\text { angular size }\left({ }^{\prime \prime}\right)\end{array}$ \\
\hline STIS open mode & 550 & 441 & 25900 & 0.065 & 0.025 \\
NICMOS F110W & 1132 & 588 & 108539 & 0.23 & 0.075 \\
NICMOS F160W & 1608 & 399 & 128441 & 0.23 & 0.075 \\
\hline
\end{tabular}

mainly by cosmologic dimming of surface brightness by a factor of $(1+z)^{4}$.

While there is a growing inflow of data concerning general statistical properties of high redshift galaxies, just a few papers have concentrated on the internal properties of distant disk galaxies, e.g. kinematics, detailed surface brightness and color distribution. For example, rotation curves for distant disk galaxies $(z \leq 1)$ have been measured by Vogt et al. (1996, 1997), Erb et al. (2003) and Böhm et al. (2004). Moth \& Elston (2002) have studied HDF-N field and constructed rest-frame $\left(U V_{218}-U_{300}\right)$ color profiles and rest-frame $B$ surface brightness profiles for 83 galaxies at $z=0.5-3.5$. They report color gradient $\Delta\left(U V_{218}-U_{300}\right) / \Delta r$ rising with redshift, which suggests that star formation has shifted inwards during the evolution of galaxies.

In Tamm \& Tenjes (2003, 2005), we combined luminosity distribution with rotation curves to construct self-consistent mass distribution models for disk galaxies out to $z \approx 1$. We noted that for all the 7 galaxies studied, the usual exponential disk assumption gave poor fit to the luminosity profiles; a more steep cut-off at outer radii, provided by the Sérsic (1968) index $n<1$, was needed. Was this because of selection effects, evolution of disk parameters with time or anything else? With this question in mind, in the present paper we concentrate on the luminosity and color profiles of disk galaxies at a redshift range as wide as possible, in an attempt to detect evolutionary effects of several disk parameters with a particular emphasis on the shape of the profiles. To achieve this, our main task is to acquire surface brightness profiles as well as color profiles for a sample of disk galaxies, introducing as few selection effects as possible.

We have chosen a small field in the southern sky, the Hubble Deep Field (HDF) South NICMOS parallel field for the study. In addition to the information offered by the multicolor observations of this field, the choice of the southern sky provides comparison with the well-studied HDF-N region.

In the present work we take $H_{0}=65 \mathrm{~km} \mathrm{~s}^{-1} \mathrm{Mpc}^{-1}$ and $\Omega_{0}=1, \Omega_{\mathrm{m}}=0.3, \Omega_{\Lambda}=0.7$.

\section{Observations and sample selection}

To avoid generation of artificial evolutionary trends, galaxies at different redshifts should be observed in the same restframe waveband. This precaution eliminates possible effects of "morphological k-correction", i.e. the dependence of the photometric properties of a galaxy on the rest-frame waveband. This phenomenon is especially frequent in the case of mid-type galaxies (Papovich et al. 2003). For comparison with near-by galaxies, rest frame optical passband would be the best choice. To study rest-frame optical properties of galaxies at high redshifts, observations made in near infrared are required. Presently, the best available resolution in near infrared is offered by the NICMOS camera aboard the Hubble Space Telescope (HST). However, the imaging properties of the NICMOS camera are still far behind those of the HST optical cameras.

During HDF South observations (Williams et al. 2000), the NICMOS camera was pointing in a slightly different direction, and in this way, a parallel field was created with NIC-3 camera through the $J, H$ and $K$ broad-band infrared filters. This field is also covered by WFPC2 camera $I$ filter observations of Flanking Field 9 (Lucas et al. 2003). Additionally, the NICMOS parallel field has been observed with the HST STIS camera using the open mode, i.e. without any filter, which gives a very broad bandwidth with the central wavelength matching that of the standard $V$-band. Regrettably, the $I$ and $K$ band observations could not be used here - the former suffers from insufficient depth and the latter has too high noise and background level (the $K$ images were taken during "bright" time, i.e. when the telescope was pointed near the bright limb of the earth).

Observations conducted in the three remaining passbands $-V, J$ and $H$ - have been used in the present study. The basic properties of these observations and the final images are given in Table 1.

We have used the fully calibrated, combined and dithered images of the HDF-S NICMOS parallel field, available via the homepage of the Space Telescope Science Institute. The lower limit of detectable surface brightness on these exposures is approximately $27.5 \mathrm{mag} / \operatorname{arcsec}^{2}$.

Table 1 shows that on NICMOS exposures the full-weight half-maximum (FWHM) of the point-spread function (PSF) is $0.23^{\prime \prime}$, causing serious distortion of the galactic images. The width of the PSF as well as its slightly non-circular shape cause uncertainties in deconvolution of the images.

Unfortunately, spectroscopic redshifts of the galaxies in the HDF-S NICMOS parallel field have not been measured. Photometric redshifts, based on 9-band measurements, have been calculated by Yahata et al. (2000).

Galaxies with redshift $z>0.5$ were selected for the further study. Discrimination between Hubble types is not always a simple task at high redshifts. Many objects may be in transition stages (protodisks or protospheroids) even at $1<z<2$ (Conselice et al. 2004). Morphological structure of these objects was inspected visually on the basis of $H$-band exposures, all galaxies suspected for being disks were included in the 
sample. In some cases, also the high-resolution STIS $V$-band images were checked (see Table 1 for the comparison of PSFs and projected pixel sizes). Final confirmation for the sample to consist of disk galaxies comes from the luminosity profiles, which all exhibit Sérsic index $n<2$ and should thus have a latetype morphology (Andredakis et al. 1995; Ravindranath et al. 2004).

Galaxies showing significant asymmetry or irregular shape were excluded, because fitting ellipses to their isophotes would give rather scattered light profiles (light distribution is very sensitive to the galactic center position) and their interpretation would not be straightforward in the context of the present models and subsequent analysis. Thus possible starburst and interacting galaxies were rejected as photometrically incomparable to regular disks.

These selection criteria finally set a redshift limit at $z=$ 2.6, beyond which no disk galaxies could be distinguished with acceptable confidence. A final sample of 22 galaxies qualified for further analysis.

The choice of passbands described above allows us to determine rest-frame optical luminosity profiles, using STIS observations for redshifts $z<1.0$ and NICMOS $J$ and $H$ observations for $1.1<z<2.0$ and $z>2.1$, respectively; the mean central rest-frame wavelength thereby becomes $420 \mathrm{~nm}$, corresponding to Johnson $B$ filter. Color information can be obtained, using STIS and NICMOS $J$ observations at $z<1.1$, and NICMOS $J$ and $H$ bands at $z>1.4$ (no galaxies were found at $1.1<z<1.4$ ). The mean central rest-frame wavelengths thus become $350 \mathrm{~nm}$ and $580 \mathrm{~nm}$, allowing the derivation of $(U-V)$ color distribution. Note that here STIS observations are used for $U$ and $B$ rest-waveband photometry for the same galaxies. This can be justified by the very wide "passband" of STIS open mode, depending only on the detector sensitivity. A drawback of using such a wide wavelength range lies in the danger of suppressing possible color-features and trends, but it does not introduce or artificially amplify the evolutionary effects.

The STIS and NICMOS $J$-band images of the galaxies are presented in Fig. 1. STIS images are designated as $V ; x$ and $y$ pixel coordinates of the galaxies on NICMOS images are used as galactic names; $z$ is photometric redshift. Where necessary, the galaxies of the present sample have been encircled to avoid confusion. Some of the sample galaxies lie at the edge of the field of view of the STIS camera and the galaxy 380-1027 falls just outside it, thus no color-information could be acquired in the latter case.

\section{Data reduction and photometry}

To determine absolute magnitudes and especially to generate color profiles, it is vital to precisely estimate the background level for each galaxy. We measured background level at 2-4 empty-looking fields around each galaxy and found the uniformly subtracted background levels of both STIS and NICMOS final images to be slightly over-estimated. Individual background estimates for each galaxy were necessary due to considerable variations within each passband image, ranging from -0.00015 to 0 counts per second in the case of STIS and from -0.00005 to 0.00002 counts per second in the case of NICMOS images.

Before fitting ellipses to the galactic images with the STSDAS task ELLIPSE, we deconvolved images with PSF. Correct deconvolution is very important, if one wishes to conduct analysis of the luminosity and color profiles of faint objects. The bulge component of a disk galaxy often acts as a point source at high redshifts; wrong estimating of the spreading of its flux may cause significant drift of the parameters of the disk component.

In the case of the NICMOS images, the model PSFs were created using stellar images found in the same field. In the case of STIS, no unsaturated stellar images were found and the model PSF was created using the TinyTim package.

Ellipses were interactively fitted to the isophotes. Any object, which did not seem to be part of a given galaxy, was masked. Both fixed and free wandering values were tested for ellipse centers; the resulting differences in surface brightness profiles are included in the uncertainties of the profiles in Fig. 2. Ellipticity and position angle were kept fixed according to the outer isophotes of the galaxies.

For rest-frame photometry we transformed the observed passbands into rest-frame standard passbands, which needed minimal k-correction after redshifting. $B$-profiles and $(U-$ $V$ ) color profiles were found to be most suitable (see Sect. 2).

No reliable k-corrections existed for the transformations from neither the STIS open mode nor the NICMOS passbands and we had to calculate them. In our calculations we relied on the synthetic spectra of redshifted Sb galaxies (as a mid-way between $\mathrm{S} 0$ and $\mathrm{Sc}$ galaxies) constructed by Bicker et al. (2004) according to their chemical evolution models. In these spectra, effects of evolution and redshifting had already been taken into account (therefore the correction actually includes also evolution correction). Our share was to calculate the relation between the observed flux and the redshifted standard $U, V$ or $B$ filter flux for each set of cameras, filters and redshifts. The throughput curves of the NICMOS filters and STIS clear imaging were taken from NICMOS and STIS Instrument Handbooks, respectively. Due to the available choice of filters, the final k-corrections for both rest-frame $B$-band and $(U-V)$ color remained rather modest, typically around $0.4-0.7 \mathrm{mag}$.

The final, k-corrected surface brightness profiles in restframe $B$-color with estimated error bars are presented in the upper panels of Fig. 2. In the lower panels, k-corrected rest-frame $(U-V)$ profiles and corresponding error bars are given. To calculate the extent of the error bars, inaccuracy of our Hubble type classification and the population synthesis models were taken into account. The latter was difficult to estimate precisely because no actual uncertainties of chemical evolution models are available. Thus we made a rough estimate on the basis of deviations between synthetic spectra calculated by different authors. These uncertainties are amplified by different widths and shapes of the filter passbands. The uncertainties of the synthetic spectra at higher redshifts are larger than at lower redshifts, but the estimated errors remain comparable $(0.25-0.45 \mathrm{mag})$. This is because of the spectra being stretched as $(1+z)$ with respect to the filter passband on one hand, thus enabling more exact 


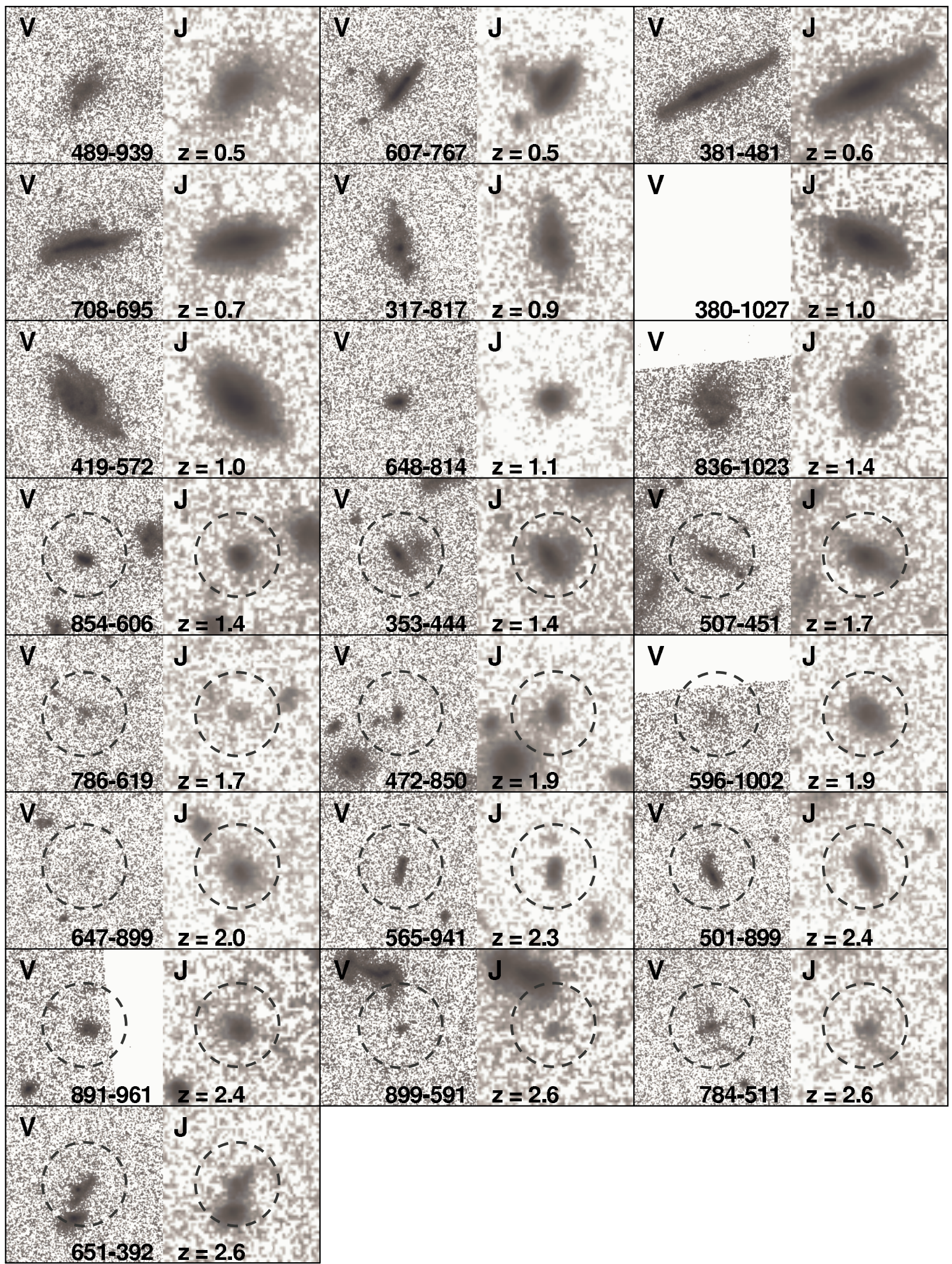

Fig. 1. Galaxies studied in the present paper as they appear on STIS open mode $(\sim V)$ and NICMOS $J$ passband images of the HDF-S NICMOS field. For clarity, the studied galaxy has been encircled on some images. The angular size of each image is $4.5 \times 4.5$ arcsec. $x$ and $y$ coordinates of NICMOS images are used as galactic names; $z$ is photometric redshift. 

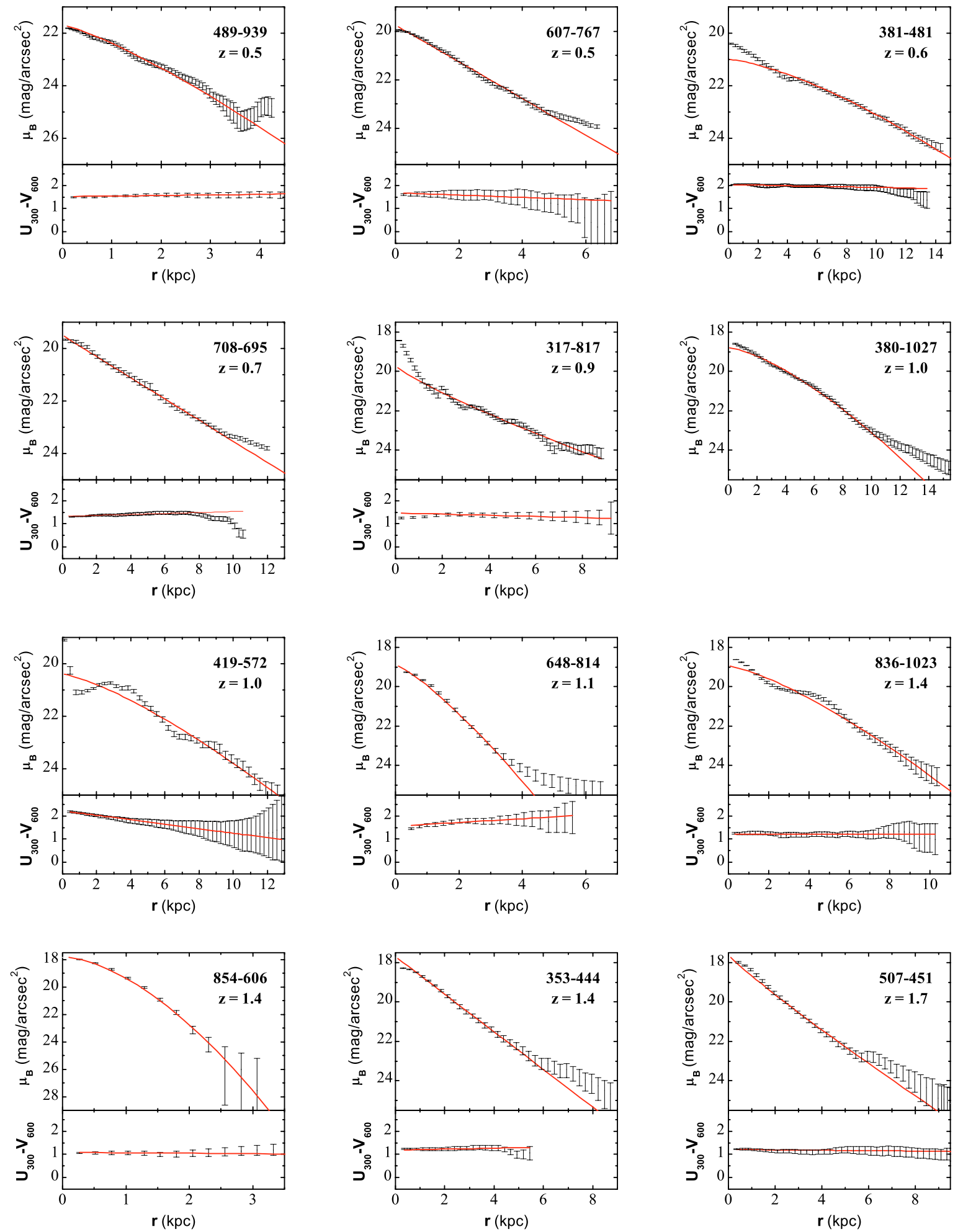

Fig. 2. Rest-frame $B$-band luminosity profiles with Sérsic fits, and rest-frame $(U-V)$ color profiles with linear fits.

determination of the flux, and the usage of the wide STIS imaging throughput at the tricky $U V$-region for lower redshift galaxies on the other hand, which can not be very accurate. The step of determining actual flux according to the synthetic spectra dominates in the estimates of the uncertainties of color measurements in Fig. 7.
The usage of different filters and detectors for imaging at different redshifts would cause errors in absolute photometry and introduce artificial trends with respect to redshift, if calibrations or $\mathrm{k}$-corrections were calculated improperly. Considerable errors of this kind would be seen as a jump in magnitude-redshift plots at the redshift of the filter change, 

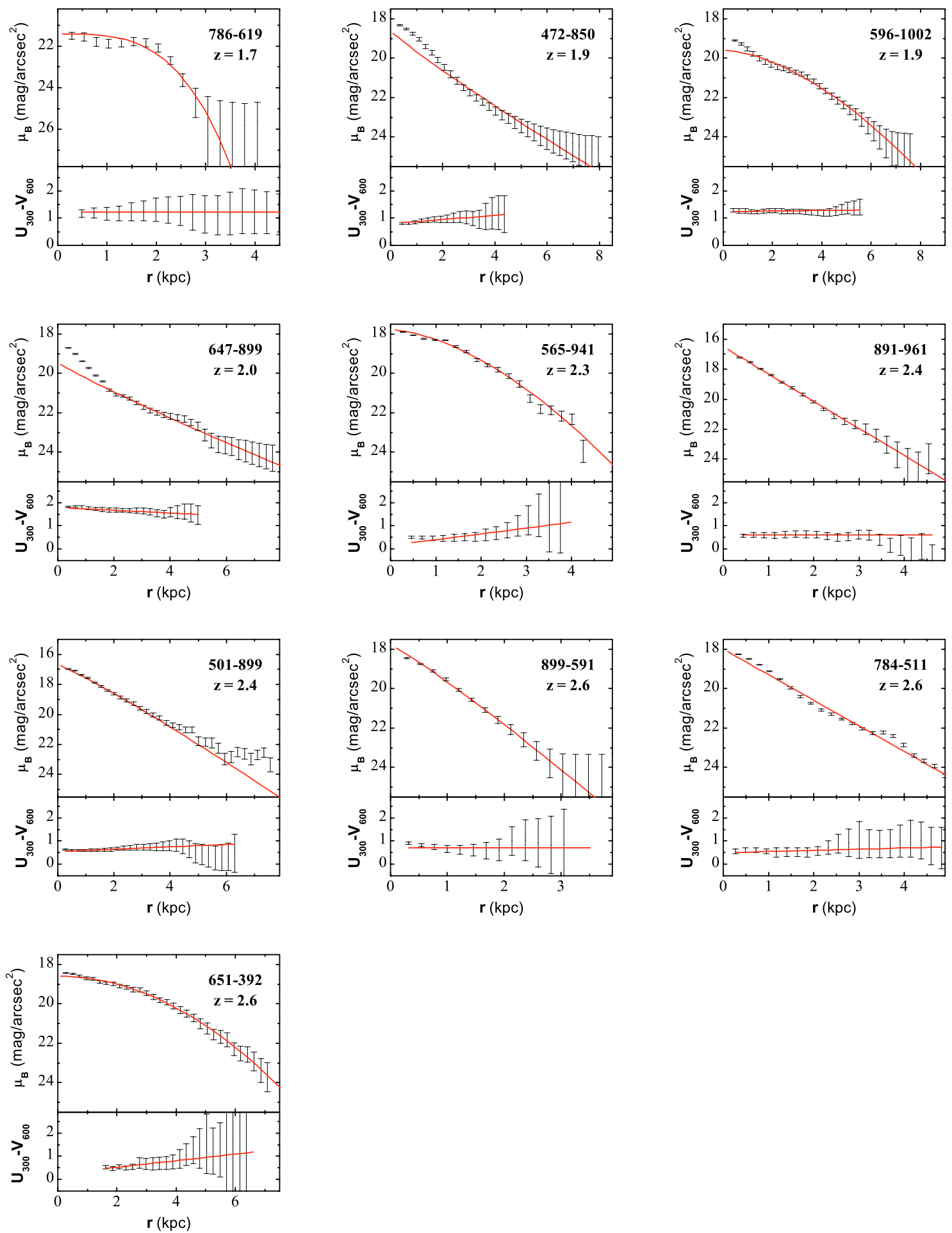

Fig. 2. continued.

e.g. between $z=1.1$ and $z=1.4$ in our case of $(U-V)$ vs. $z$ graph in Fig. 5. The graph of absolute $B$ magnitudes $M_{B}$ vs. $z$ was also checked for this effect; no jumps were found.

\section{Fitting by model profiles}

Although in some cases (usually at smaller redshifts), the bulge component was distinguishable from the disk, no attempt was made to split the luminosity profile into two components, because this would have given systematically different disk parameters at different redshifts.

Luminosity profiles were fitted by a Sérsic surface density distribution (Sérsic 1968):

$$
I(a)=I(0) \exp \left[-b_{n}\left(r / r_{\mathrm{e}}\right)^{1 / n}\right]
$$

In this formula, $r$ is the distance along the galactic major axis, $r_{\mathrm{e}}$ is the effective radius, containing half of the total luminosity, 


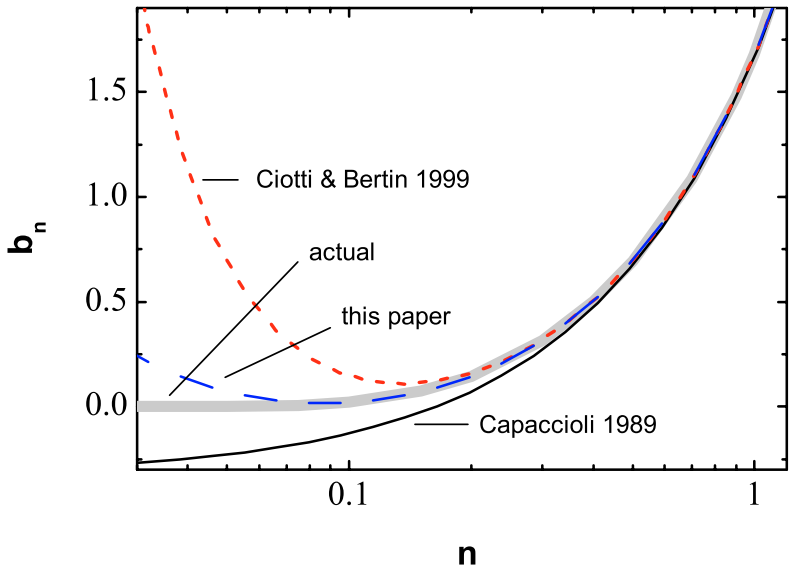

Fig. 3. Normalizing parameter $b_{n}$ in Sérsic formula (1) at low $n$ values. Comparison of our approximation (2) with numerically calculated actual values, together with approximations by Capaccioli (1989) and Ciotti \& Bertin (1999).

$n$ is the shape parameter, setting the curvature of the profile, and $b_{n}$ is a normalizing constant, dependent on $n$, enabling to keep $r_{\mathrm{e}}$ the half-light radius. To determine $b_{n}$, either gamma functions or integral equations have to be numerically solved. Usually, $b_{n}=1.9992 n-0.3271$ (Capaccioli 1989) or other formulae are used (e.g. Prugniel \& Simien 1997; Moriondo et al. 1998; Ciotti \& Bertin 1999), which all can be successfully applied at $n$ values above $n \approx 0.5$. If lower $n$ values are present, these solutions start to mislead. Therefore, we have calculated our own approximation,

$b_{n}=2 n-\frac{1}{3}+\frac{1}{65 n}$,

which can well be used down to $n \approx 0.1$. Comparison of our approximation (2) with numerically calculated values together with approximations by Capaccioli (1989) and Ciotti \& Bertin (1999) is given in Fig. 3. To emphasize deviations at small $n$ values, logarithmic scale is used.

As a tool for fitting surface brightness distribution, the Sérsic law has a purely empiric background. Starting from a spatial density distribution law and projecting it along the line of sight would be a more physical method, also allowing a straightforward comparison with kinematic data and thereby enabling the construction of self-consistent models for mass distribution. Thus, fitting of a surface density distribution, deduced from the space density distribution law of the very general form

$\rho(a)=\rho(0) \exp \left[-\left(a /\left(k a_{0}\right)\right)^{1 / n}\right]$

was also tested for comparison. Here, $\rho(0)$ is the central density, $a=\sqrt{R^{2}+z^{2} / q^{2}}$, where $R$ and $z$ are two cylindrical coordinates and $q$ is the axial ratio; $a_{0}$ is the characteristic radius and $k$ is a normalizing parameter, similar to Sérsic $b_{n}$ (see Einasto \& Haud 1989; and Tenjes et al. 1994, for more details about this distribution and reasoning for its usage). This method gives the luminosity profile a somewhat different shape, but if fitted to a real density distribution, the main parameters $\left(n, a_{0} \approx r_{\mathrm{e}}\right)$ remain similar to those acquired from the Sérsic law.

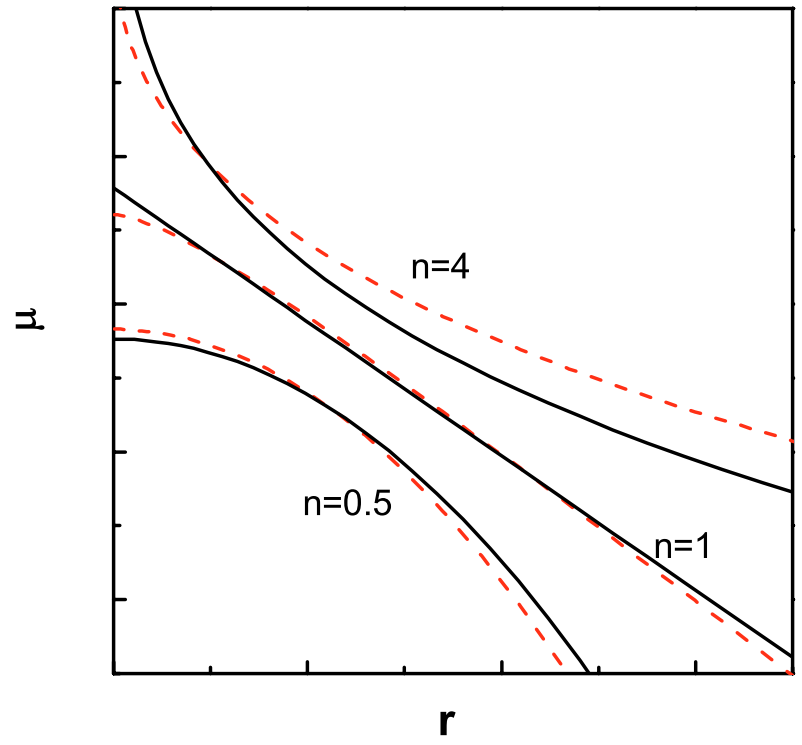

Fig. 4. Comparison of the Sérsic surface brightness distribution (solid lines) and a distribution, derived from the space density distribution of Eq. (3) (dashed lines) at different $n$ values. Surface brightness value $\mu$ and radius $r$ are given in the same relative units for both distributions. No rescaling has been conducted to match the curves.

Comparison of these two distributions at different $n$ values is given in Fig. 4. Surface brightness value $\mu$ and radius $r$ are given in the same relative units for both distributions. We stress that no rescaling has been conducted for matching the distributions; the fit has been achieved by keeping the total luminosities equal. Note that a perfectly exponential distribution, corresponding to the Sérsic law with $n=1$ cannot be attained with distribution (3).

For galaxies in the present sample, no additional kinematic data are available at present. For this reason, we only present here the parameters resulting from fitting of the Sérsic law (1) to the brightness profiles. This also allows more direct comparison with other studies.

\section{Results and discussion}

The rest-frame $B$ luminosity profiles, fitted to the Sérsic law, and the $(U-V)$ color profiles with linear fits are shown in Fig. 2. The main properties of the galaxies and the results of the photometric analysis are given in Table 2. Distribution of the parameter $n$, the effective radius $r_{\mathrm{e}}$, the $(U-V)$ color distribution, and color gradient $\Delta(U-V) / \Delta r$ are shown in Figs. 5-7 as a function of redshift. The three different brightness levels of the data points indicate three absolute rest-frame $B$ magnitudebins of the galaxies: white points stand for galaxies with $M_{B}<$ $-20 \mathrm{mag}$, grey points for $M_{B}=-19 \ldots-20 \mathrm{mag}$ and black points for $M_{B}>-19 \mathrm{mag}$. For equal treatment of the galaxies at different redshifts, absolute brightness has been calculated within rest-frame surface brightness $\mu_{B}<24 \mathrm{mag} / \operatorname{arcsec}^{2}$. 
Table 2. General galactic parameters.

\begin{tabular}{|c|c|c|c|c|c|c|c|c|}
\hline Name $^{a}$ & $\begin{array}{l}\text { NED name } \\
\text { (also J2000 coordinate) }\end{array}$ & $z^{b}$ & $\begin{array}{l}M_{B} \\
(\mathrm{mag})\end{array}$ & $\begin{array}{l}L_{B} \\
10^{10} L_{\odot}\end{array}$ & $\begin{array}{l}r_{\mathrm{e}} \\
(\mathrm{kpc})\end{array}$ & $n^{c}$ & $\begin{array}{l}U-V \\
(\mathrm{mag})\end{array}$ & $\begin{array}{l}\Delta(U-V) / \Delta r \\
(\mathrm{mag} / \mathrm{kpc})\end{array}$ \\
\hline 489-939 & HDFS J223252.31-603827.3 & 0.49 & -16.4 & 4.3 & 1.8 & 0.8 & 1.55 & 0.03 \\
\hline $607-767$ & HDFS J223251.11-603840.2 & 0.51 & -17.7 & 3.3 & 2.3 & 1 & 1.40 & -0.05 \\
\hline $381-481$ & HDFS J223253.42-603901.6 & 0.6 & -18.8 & 3.2 & 6.7 & 0.7 & 1.66 & -0.01 \\
\hline $708-695$ & HDFS J223250.08-603845.6 & 0.66 & -19.3 & 3.2 & 4.5 & 1 & 1.26 & 0.02 \\
\hline $317-817$ & HDFS J223253.65-603846.9 & 0.89 & -18.3 & 1.9 & 3.0 & 1.2 & 1.28 & -0.01 \\
\hline $380-1027$ & HDFS J223253.43-603820.6 & 0.99 & -20.8 & 1.8 & 4.1 & 0.7 & - & - \\
\hline $419-572$ & HDFS J223253.03-603854.8 & 1.01 & -20.1 & 1.7 & 4.8 & 0.75 & 1.69 & -0.10 \\
\hline $648-814$ & HDFS J223250.70-603836.6 & 1.12 & -18.6 & 1.6 & 1.3 & 0.8 & 1.66 & 0.08 \\
\hline $836-1023$ & HDFS J223248.78-603821.0 & 1.37 & -21.1 & 1.3 & 3.5 & 0.75 & 1.18 & -0.01 \\
\hline $854-606$ & HDFS J223248.59-603852.2 & 1.37 & -19.6 & 1.2 & 0.7 & 0.6 & 1.05 & -0.02 \\
\hline $353-444$ & HDFS J223253.71-603904.4 & 1.39 & -20.1 & 1.2 & 1.9 & 1 & 1.22 & 0.02 \\
\hline $507-451$ & HDFS J223252.13-603903.9 & 1.65 & -20.2 & 1.0 & 2.0 & 1.1 & 1.14 & -0.01 \\
\hline $786-619$ & HDFS J223249.29-603851.2 & 1.65 & -17.2 & 0.8 & 1.4 & 0.3 & 1.41 & 0.00 \\
\hline $472-850$ & HDFS J223252.49-603833.9 & 1.89 & -19.3 & 0.8 & 2.0 & 1.1 & 0.95 & 0.09 \\
\hline $596-1002$ & HDFS J223251.22-603822.6 & 1.93 & -20.0 & 0.8 & 2.6 & 0.6 & 1.23 & 0.01 \\
\hline $647-899$ & HDFS J223250.70-603830.3 & 2.01 & -19.7 & 0.8 & 2.8 & 1.1 & 1.71 & -0.06 \\
\hline $565-941$ & HDFS J223251.54-603827.1 & 2.33 & -19.7 & 0.5 & 1.6 & 0.6 & 0.64 & 0.25 \\
\hline $501-899$ & HDFS J223252.19-603830.2 & 2.39 & -20.8 & 0.4 & 1.7 & 0.9 & 0.64 & 0.05 \\
\hline $891-961$ & HDFS J223248.22-603825.6 & 2.39 & -20.8 & 0.3 & 1.0 & 1 & 0.61 & 0.00 \\
\hline $899-591$ & HDFS J223248.13-603853.4 & 2.55 & -19.3 & 0.2 & 0.9 & 0.9 & 0.62 & 0.00 \\
\hline $784-511$ & HDFS J223249.30-603859.4 & 2.56 & -19.2 & 0.1 & 1.5 & 1 & 0.58 & 0.05 \\
\hline $651-392$ & HDFS J223250.67-603908.3 & 2.58 & -19.8 & 0.1 & 2.7 & 0.5 & 0.59 & 0.20 \\
\hline
\end{tabular}

${ }^{a}$ The $x$ and $y$ pixel coordinate on HDF-S NICMOS final images.

${ }^{b}$ Photometric redshifts are from Yahata et al. (2000).

${ }^{c}$ Steepness parameter of the best-fit Sérsic profile.

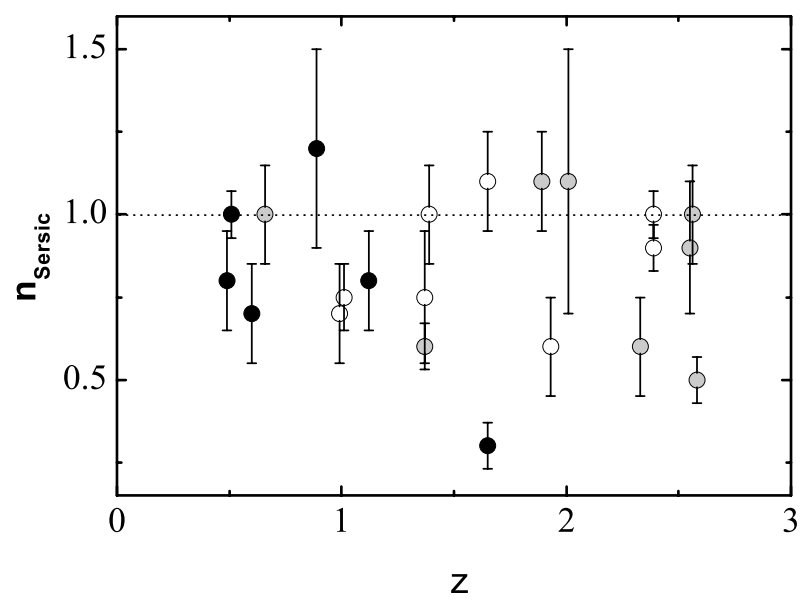

Fig. 5. The Sérsic luminosity profile curvature $n$ as a function of redshift. Open circles stand for galaxies with absolute luminosity $M_{B}<-20 \mathrm{mag}$, gray circles for $-19 \mathrm{mag}<M_{B}<-20 \mathrm{mag}$ and black circles for $M_{B}<-19$ mag.

\subsection{The shape of the luminosity profiles}

As is evident from the present sample, nearly half of galaxies cannot be fit to an exponential disk model, while for the rest, $n \approx 1$ works well out to the highest redshifts (Fig. 5). A similar result was obtained by Moth \& Elston (2002) for the HDF North field. As suggested in Tamm \& Tenjes (2005), the more curved profiles might indicate the effect known as disk truncation, discovered by studies of local edge-on disk galaxies (de Grijs et al. 2001; Pohlen et al. 2002) and more recently detected also at higher redshifts (Pérez 2004; Trujillo \& Pohlen 2005). Such a luminosity distribution is sometimes fitted by a double-exponential profile (Pohlen et al. 2002; Pérez 2004). The physical background of disk truncation has not become clear as yet (see e.g. Sasaki 1987; and de Grijs et al. 2001, for more discussion about the origin of this phenomenon).

In contrast to these truncated disks, some galaxies in the present sample have flattening profiles (e.g. 607-767, $708-695,380-1027,353-444)$. Similar profiles have been measured near $z=1$ for gamma-ray burst-selected disks (Conselice et al. 2005b) and out to $z=3$ for large disks in HDF-South primary field (Labbé et al. 2003). Erwin et al. (2005) have found a significant proportion of local barred S0-Sb galaxies with flattening profiles, which they call "antitruncated" disks. They claim that, at least in several cases, this effect might be caused by interactions. Observations of interacting galaxies (Chitre \& Jog 2002) and numeric simulations 


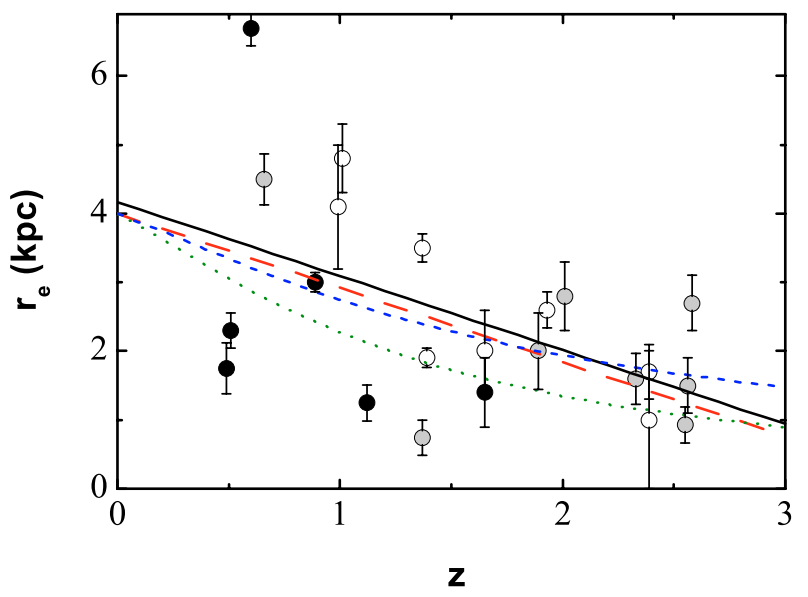

Fig. 6. The Sérsic luminosity profile effective radius $r_{\mathrm{e}}$ as a function of redshift. Open circles stand for galaxies with absolute luminosity $M_{B}<-20$ mag, gray circles for -19 mag $<M_{B}<-20$ mag and black circles for $M_{B}<-19 \mathrm{mag}$. The solid line shows a least-squares linear fit to the current sample $r_{\mathrm{e}}(z) / r_{\mathrm{e}}(0)=1-0.26 z$; the long-dashed line is the relation $r(z) / r(0)=1-0.27 z$ derived by Bouwens \& Silk (2002); the dotted and short-dashed lines give the relations $r_{\mathrm{e}} \sim H(z)^{-1}$ and $r_{\mathrm{e}} \sim H^{-2 / 3}(z)$, respectively.

(Bournaud et al. 2005) have demonstrated the possibility of mergers to smear out the outer parts of luminosity profiles. In the case of our sample, visual companions are present for all four galaxies with flattened profiles, thus interactions may have a role in the development of this flattening. The companions have been masked prior to ellipse fitting, therefore the luminosity of the companions cannot be responsible for the effect.

The 7 galaxies studied in Tamm \& Tenjes (2003, 2005) have significantly low $n$ values compared to the present sample. This is most likely a result of the small size of the samples or selection effects, favoring luminous galaxies with regular kinematics.

\subsection{Sizes of disks $r_{e}$}

In Fig. 6, evolution of the size of the present sample is shown. Error bars of sizes were derived by varying $r_{\mathrm{e}}$ while fitting the Sérsic law within the surface brightness profile error bars. The slight degeneracy between parameters $n$ and $r_{\mathrm{e}}$ has also been taken into account.

Starting from the locally observed Universe and modeling galactic evolution backwards, Bouwens \& Silk (2002) derived a scaling relation $r(z) / r(0)=1-0.27 z$ for the $B$-band radius. The long-dashed line in Fig. 6 shows this relation. The leastsquares linear fit to our sample gives $r_{\mathrm{e}}(z) / r_{\mathrm{e}}(0)=1-0.26 z$ (solid line). Here, the median value of Sérsic half-light radii $\left\langle r_{\mathrm{e}}\right\rangle \approx 4.2 \mathrm{kpc}$ for late-type galaxies from the SDSS survey (Shen et al. 2003) has been used as the value of $r_{\mathrm{e}}(0)$ (considering the median absolute luminosity of the present sample). It is seen that the model developed by Bouwens \& Silk (2002) fits our data rather closely. Our result is consistent with the small $r_{\mathrm{e}}$ values found for UDF galaxies by Elmegreen et al. (2005).

Galactic size evolution has also been predicted from simple models of the hierarchical structure formation scenario

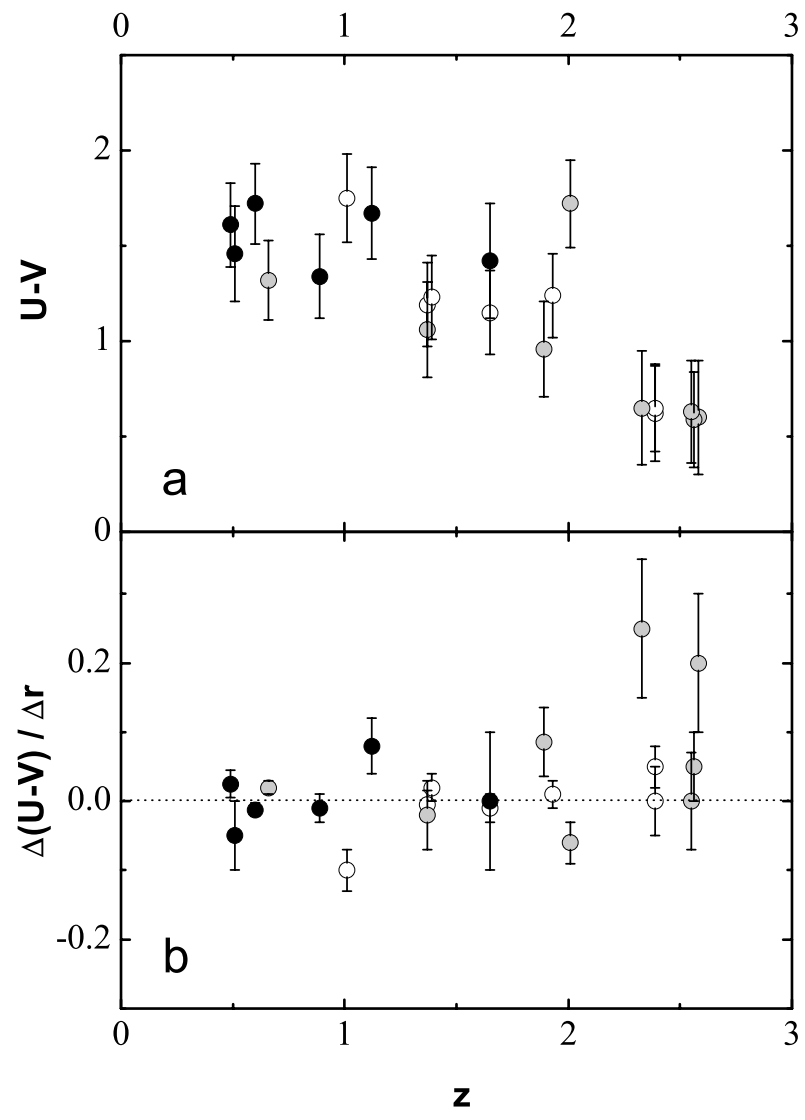

Fig. 7. Galactic parameters - rest frame $(U-V)$ color a) and $\Delta(U-$ $V) / \Delta r \mathbf{b})-$ as a function of redshift. Designations are the same as in Fig. 6.

and an approximate scaling has been derived with disk formation time: $r_{\mathrm{e}} \sim V_{\mathrm{vir}} / H(z)$, where in the case of $\Omega_{0}=1$, $H(z)=H_{0}\left[\Omega_{\mathrm{m}}(1+z)^{3}+\Omega_{\Lambda}\right]^{1 / 2}$ (Mo et al. 1998). In a study of galaxies at a redshift range $z=1.5-5$, Ferguson et al. (2004) found the scaling of $r_{\mathrm{e}} \sim H^{-1}(z)$ to give a good fit to their sample, while Trujillo et al. (2005) achieved a better fit with $r_{\mathrm{e}} \sim H^{-2 / 3}(z)$ for galaxies at $z=0.3-2.5$. The relations $r_{\mathrm{e}} \sim H^{-1}(z)$ and $r_{\mathrm{e}} \sim H^{-2 / 3}(z)$, scaled with $r_{\mathrm{e}}(0) \approx 4 \mathrm{kpc}$, are also shown in Fig. 6, as dotted and short-dashed lines, respectively. The former shows a decrease significantly faster than that of the present sample. However, it must be kept in mind that the theoretical relation uses the disk formation redshift instead of the observed redshift for scaling. Further more, in contrast to the present sample and the literature referred to above, Ravindranath et al. (2004) and Cassata et al. (2005) did not detect any significant evolution of galaxy sizes with redshift in a thorough study of galaxies at $z \leq 1.2$.

Figure 6 shows that the trendline fitted to the present sample is quite sensitive to the data point corresponding to the largest galaxy with $r_{\mathrm{e}}=6.7 \mathrm{kpc}$ at $z=0.6$. Its extensive disk is obvious also on Fig. 1 and its light profile is a very regular one, thus there is no doubt that this galaxy is clearly a large disk galaxy. The only reason for this data point to be significantly offset could be a wrong photometric redshift estimate. 


\subsection{Color $(U-V)$ and color gradient $\Delta(U-V) / \Delta r$}

In Fig. 7a, rest frame $(U-V)$ color as a function of redshift is presented. The $(U-V)$ color shows a mild decrease until $z \approx 2$, followed by a notable drop of roughly 0.5 mag by $z \approx 2.5$. At lower redshifts, the dependence can be compared to the Deep Groth sample studied by Weiner et al. (2005), for which a very slight, if any, decrease of $(U-B)$ was detected for late-type galaxies in the redshift range $z=0-1.5$. The notable drop of the $(U-V)$ values at $z \approx 2$ cannot be due to miscalibration a uniform set of filters and calibrations has been used for the redshift range $z=1.4-2.6$ (see Sects. 2 and 5.5). The drop can rather be related to a major star formation peak at $z>2$ (see below). A decrease of the rest-frame $(U-V)$ color with increasing redshift up to $z \sim 3$ was derived also by Kajisawa \& Yamada (2005). More detailed comparison with our results is not possible at present because in their study, different morphological types are presented together. Our result is quantitatively close also to the result obtained from the analysis of the synthetic spectra of Bicker et al. (2004). Absolute $(U-V)$ values are rather sensitive to k-correction, which, in turn, is uncertain. This may cause a constant vertical shift of $(U-V)$ values. However, trends in Fig. 7 should be independent of these uncertainties.

Figure $7 \mathrm{~b}$ shows that at redshifts $z \leq 2$, there are small or no color gradients. Gradients begin at $z>2$ and are dominantly positive. A similar rise with redshift of the rest-frame color $\left(U_{218}-U_{300}\right)$ gradients was discovered by Moth \& Elston (2002), suggesting that star-formation was more centrally concentrated at higher redshifts.

Recent hydrodynamical simulations of galaxies in $\Lambda$ CDM cosmology by Robertson et al. (2004) indicate that star formation in disks peaks between redshifts $z=2-4$. Studies of the Fundamental Plane of early-type galaxies at redshifts $z \leq 1.3$ have also shown that the last epoch of major star formation peaks at $z \sim 2-3.5$ (see Holden et al. 2005, and references therein). A jump in star formation beyond redshift $z=2$ matches well with the jump in $(U-V)$ color in Fig. 7a (see above).

Smaller color gradients at lower redshifts $z=0.5-2$ are due to smoothing by interactions in disk evolution (Conselice et al. 2005a) and large scale gas motions (Samland \& Gerhard 2003).

Positive color gradients also exist in local disk galaxies (MacArthur et al. 2004). Positive color gradients may indicate disk formation from inside out. For the Milky Way protogalaxy such formation scenario was suggested by van den Bergh (1993). In the case of high-redshift galaxies, direct interpretation of color gradients may be rather complicated. Color gradients are determined by radial distribution of initial metallicities, stellar ages, star-formation rate, gas accretion details etc. Dependently on model details, both outside-in and insideout formation of disks can be simulated (Sommer-Larsen et al. 2003; Robertson et al. 2004).

According to the analysis of their disk galaxy formation model, Westera et al. (2002) have found that luminosity from $U$ to $V$ mainly indicates star formation, and the effects of age and metallicity are negligible. Thus the positive color gradient most

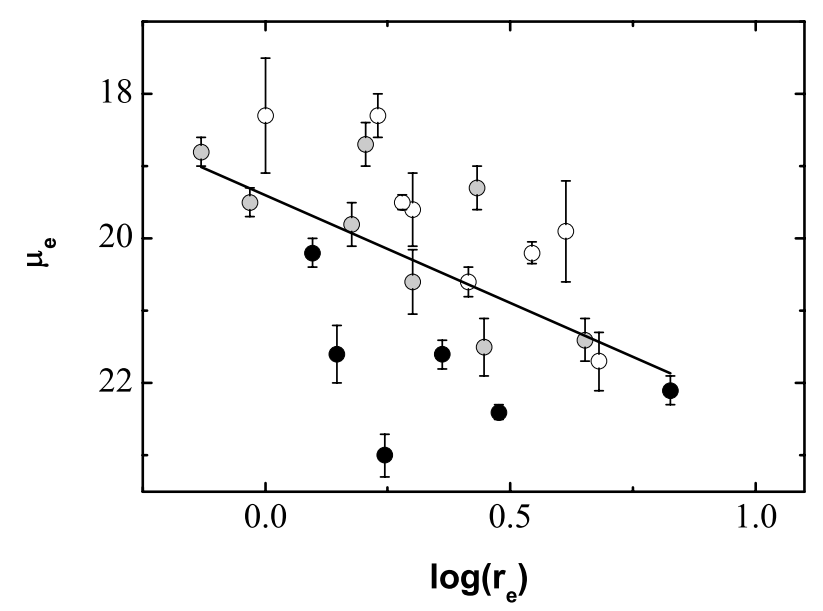

Fig. 8. Effective surface brightness $\mu_{\mathrm{e}}$, plotted as a function of $\log \left(r_{\mathrm{e}}\right)$. Designations are the same as in Figs. 5, 6. Solid line shows a leastsquare fit.

possibly refers to intensive star formation in central parts of galaxies at $z>2$.

Relying on $(U-V)$, we may roughly estimate the disk formation time. According to our photometry, the disks at $z \sim 2.5$ have $(U-V) \sim 0.7$. On the basis of simple stellar population chemical evolution models (Worthey 1994; Bressan et al. 1994), these disks have ages 1-2 Gyr and have thus formed at $z=3.5-7$.

\subsection{Surface brightness at effective radius}

Coenda et al. (2005) have shown that disks obey similar photometric scaling relations as spheroids and elliptical galaxies. Degeneracy exists between the effective radius, effective surface brightness, the parameter $n$ and absolute magnitude. For the current sample, a trend can be shown for the effective surface brightness $\mu_{\mathrm{e}}$ to diminish with larger effective radius $r_{\mathrm{e}}$, presented in Fig. 8. Least squares fit gives $\mu_{\mathrm{e}}=3.0 \log r_{\mathrm{e}}+$ 19.4 mag, which, regarding the small size of the sample, is remarkably close to the dependence found by Coenda et al. (2005) $\mu_{\mathrm{e}}=3.4 \log r_{\mathrm{e}}+18.7 \mathrm{mag}$.

\subsection{Uncertainties of the study}

Small sample sizes and selectional biases are critical topics for most of the studies of high-redshift galaxies. The present sample of 22 galaxies can not offer reliable statistics and cautions against jumping into severe conclusions.

Morphological identification of these disk galaxies was obtained by visual inspection of $H$-band exposures, all galaxies suspected for being disks were included in the sample. In some cases, also the high-resolution STIS $V$-band images were checked. Some of the galaxies of the present sample have been studied on the basis of NICMOS images by Rodighiero et al. (2001), and classified as E-S0. We would like to point out that for classification and accurate photometry it is important to carefully deconvolve the PSF of NICMOS images. Even in the case of good restoration of NICMOS images, additional 
observations with other detectors, preferably with higher resolution (e.g. STIS in the present case) still prove beneficial. For example, visual inspection of the STIS image of the galaxy 419-572 reveals its nature of a late-type (at least $\mathrm{Sb}$ ) galaxy.

Usually, the cosmological dimming as $(1+z)^{4}$ favors the selection of more luminous galaxies at higher redshifts. For our sample, Figs. 5-8 reveal no clear dependence of any of the parameters on the absolute luminosity of the galaxies; this fact allows us to consider the sample to be sufficiently complete for the conducted analysis.

The selection of the sample on the basis of $H$-band imaging (corresponding to $B$-band at $z>2$ ) might cause a slight bias towards bluer galaxies at higher redshifts. On the other hand, the change of the spectral energy distribution is slow between $B$ and $R$ passbands and we consider this possible selection effect not to be a reason of a color jump at $z \simeq 2$ in Fig. 7 .

For calculating k-corrections, all galaxies were assumed to be $\mathrm{Sb}$ morphological types. This approximation causes additional scatter of absolute magnitudes and colors. However, according to our estimates, the resulting uncertainties remain within 0.2 mag even in the worst cases.

Among possible sources of errors and uncertainties, the ever insufficient imaging depth and spatial resolution should be considered. With the currently available NIR imaging a dilemma remains, whether to consider faint patches around galaxies to belong to the galaxy and include them in photometry or to mask them as extraneous. This may slightly affect the luminosity distribution at the outer regions of a given galaxy and the values of the photometric parameters, especially the shape parameter $n$. In the present case, we have followed a rather conservative masking strategy, excluding most of the "suspicious" patches. A more liberal treatment would give slightly higher $n$ and $r_{\mathrm{e}}$ values and lower absolute magnitudes. The shift of these values would be rather systematic.

Insufficient spatial resolution influences the interpretation of surface brightness profiles in the central parts of galaxies. In the present study we decided not to decompose galaxies into the bulge and disk components. Otherwise, rather serious systematic differences would appear in handling of low-redshift and high-redshift galaxies. In general, decomposing light profiles into a share of a bulge and a disk would decrease the disk radius.

Acknowledgements. We would like to thank the anonymous referee for useful comments and suggestions helping to improve the paper. We acknowledge the financial support from the Estonian Science Foundation (research grant 6106). This paper is based on NASA/ESA Hubble Space Telescope NICMOS and STIS observations of the Hubble Deep Field South obtained from the data archive at the Space Telescope Science Institute. This research had made use of the NASA/IPAC extragalactic database (NED), which is operated by the Jet Propulsion Laboratory, California Institute of Technology, under contract with the NASA.

\section{References}

Abadi, M. G., Navarro, J. F., Steinmetz, M., \& Eke, V. R. 2003, ApJ, 591, 499

Andredakis, Y. C., Peletier, R. F., \& Balcells, M. 1995, MNRAS, 275, 874
Bell, E. F., Baugh, C. M., Cole, S., Frenk, C. S., \& Lacey, C. G. 2003, MNRAS, 343, 367

Bicker, J., Fritze-v. Alvensleben, U., Möller, C. S., \& Fricke, K. J. 2004, A\&A, 413, 37

Böhm, A., Ziegler, B. L., Saglia, R. P., et al. 2004, A\&A, 420, 97

Bournaud, F., Jog, C. J., \& Combes, F. 2005, A\&A, 437, 69

Bouwens, R. J., \& Silk, J. 2002, ApJ, 568, 522

Bouwens, R. J., Illingworth, G. D., Blakeslee, J. P., Broadhurst, T. J., \& Franx, M. 2004, ApJ, 611, L1

Bressan, A., Chiosi, C., \& Fagotto, F. 1994, ApJS, 94, 63

Bunker, A. J., Spinrad, H., \& Thompson, R. I. 1999, A\&AS, 194, 0403

Capaccioli, M. 1989, in The world of galaxies, ed. H. G. Corwin, \& L. Bottinelli (Berlin: Springer-Verlag), 208

Cassata, P., Cimatti, A., Franceschini, A., et al. 2005, MNRAS, 357, 903

Chen, H.-W., Marzke, R. O., McCarthy, P. J., et al. 2003, ApJ, 586, 745

Chitre, A., \& Jog, C. J. 2002, A\&A, 388, 407

Ciotti, L., \& Bertin, G. 1999, A\&A, 352, 447

Coenda, V., Donzelli, C. J., Hernan, M., et al. 2005, AJ, 129, 1237

Cole, S., Lacey, C. G., Baugh, C. M., \& Frenk, C. S. 2000, MNRAS, 319,168

Conselice, C. J., Grogin, N. A., Jogee, S., et al. 2004, ApJ, 600, L139

Conselice, C. J., Bundy, K., Ellis, R. S., et al. 2005a, ApJ, 628, 160

Conselice, C. J., Vreeswijk, P. M., Fruchter, A. S., et al. 2005b, ApJ, 633, 29

Corbin, M. R., Vacca, W. D., Ot'Neil, E., et al. 2000, AJ, 119, 1062

de Grijs, R., Kregel, M., \& Wesson, K. H. 2001, MNRAS, 324, 1074

Einasto, J., \& Haud, U. 1989, A\&A, 223, 89

Elmegreen, B. G., Elmegreen, D. M., Vollbach, D. R., Foster, E. R., \& Ferguson, T. E. 2005, ApJ, 634, 101

Erb, D. K., Shapley, A. E., Steidel, C. C., et al. 2003, ApJ, 591, 101

Erwin, P., Beckman, J. E., \& Pohlen, M. 2005, ApJ, 626, L81

Fall, S. M., \& Efstathiou, G. 1980, MNRAS, 193, 189

Ferguson, H. C., Dickinson, M., Giavalisco, M., et al. 2004, ApJ, 600, L107

Gabasch, A., Bender, R., Seitz, S., et al. 2004, A\&A, 421, 41

Giallongo, E., Salimbeni, S., Menci, N., et al. 2005, ApJ, 622, 116

Govenato, F., Mayer, L., Wadsley, J., et al. 2004, ApJ, 607, 688

Holden, B. P., Blakeslee, J. P., Postman, M., et al. 2005, ApJ, 626, 809

Ilbert, O., Tresse, L., Zucca, E., et al. 2005, A\&A, 439, 863

Kajisawa, M., \& Yamada, T. 2005, ApJ, 618, 91

Labbé, I., Rudnick, G., Franx, M., et al. 2003, ApJ, 591, L95

Lucas, R. A., Baum, S. A., Brown, T. M., et al. 2003, AJ, 125, 398

MacArthur, L. A., Courteau, S., Bell, E., \& Holtzman, J. A. 2004, ApJS, 152, 175

Mo, H. J., Mao, S., \& White, S. D. M. 1998, MNRAS, 295, 319

Moriondo, G., Giovanardi, C., \& Hunt, L. K. 1998, A\&AS, 130, 81

Moth, P., \& Elston, R. J. 2002, AJ, 124, 1886

Nagamine, K., Cen, R., Hernquist, L., Ostriker, J. P., \& Springel, V. 2005, ApJ, 627, 608

Navarro, J. F., \& White, S. D. M. 1994, MNRAS, 267, 401

Papovich, C., Giavalisco, M., Dickinson, M., Conselice, C. J., \& Ferguson, H. C. 2003, ApJ, 598, 827

Pérez, I. 2004, A\&A, 427, L17

Pohlen, M., Dettmar, R.-J., Lütticke, R., \& Aronica, G. 2002, A\&A, 392, 807

Pohlen, M., Beckman, J., Hüttemeister, S., et al. 2004, Stellar sisk truncations: where do we stand? In Penetrating bars through masks of cosmic dust, ed. D. L. Block, K. C. Freeman, I. Puerari, \& R. Gross (Kluwer) [arXiv: astro-ph/0405541]

Poli, F., Giallongo, E., Fontana, A., et al. 2003, ApJ, 593, L1

Prugniel, P., \& Simien, F. 1997, A\&A, 321, 111 
Ravindranath, S., Ferguson, H. C., Conselice, C., et al. 2004, ApJ, 604, L9

Robertson, B., Yoshida, N., Springel, V., \& Hernquist, L. 2004, ApJ, 606,32

Rodighiero, G., Franceschini, A., \& Fasano, G. 2001, MNRAS, 324, 491

Rowan-Robinson, M. 2003, MNRAS, 345, 819

Samland, M., \& Gerhard, O. E. 2003, A\&A, 399, 961

Sasaki, T. 1987, PASJ, 39, 849

Sérsic, J. L. 1968, Atlas de Galaxies Australes, Observatorio Astronomico, Cordoba, Argentina

Shen, S., Mo, H. J., White, S. D. M., et al. 2003, MNRAS, 343, 978

Sommer-Larsen, J., Götz, M., \& Portinari, L. 2003, ApJ, 596, 47

Tamm, A., \& Tenjes, P. 2003, A\&A, 403, 529

Tamm, A., \& Tenjes, P. 2005, A\&A, 433, 31

Tenjes, P., Haud, U., \& Einasto, J. 1994, A\&A, 286, 753

Trujillo, I., \& Aguerri, J. A. L. 2004, MNRAS, 355, 82
Trujillo, I., \& Pohlen, M. 2005, ApJ, 630, L17

Trujillo, I., Rudnick, G., Rix, H.-W., Labbé, I., et al. 2004, ApJ, 604, 521

Trujillo, I., Schreiber, N. M. F., Rudnick, G., et al. 2005, ApJ, submitted [arXiv: astro-ph/0504225]

van den Bergh, S. 1993, ApJ, 411, 178

van den Bergh, S., Cohen, J. G., Hogg, D. W., \& Blandford, R. 2000, AJ, 120, 2190

Vogt, N. P., Forbes, D. A., Phillips, A. C., et al. 1996, ApJ, 465, L15

Vogt, N. P., Phillips, A. C., Faber, S. M., et al. 1997, ApJ, 479, L121

Weiner, B. J., Phillips, A. C., Faber, S. M., et al. 2005, ApJ, 620, 595

Westera, P., Samland, M., Buser, R., \& Gerhard, O. E. 2002, A\&A, 389, 761

White, S. D. M., \& Rees, M. J. 1978, MNRAS, 183, 341

Williams, R. E., Baum, S., Bergeron, L. E., et al. 2000, AJ, 120, 2735

Worthey, G. 1994, ApJS, 95, 107

Yahata, N., Lanzetta, K. M., Chen, et al. 2000, ApJ, 538, 493 